\title{
Association of metabolic and genetic heterogeneity in head and neck squamous cell carcinoma with prognostic implications: integration of FDG PET and genomic analysis
}

Jinyeong Choi ${ }^{1}$, Jeong-An Gim¹', Chiwoo Oh' ${ }^{1}$, Seunggyun Ha ${ }^{2,3}$, Howard Lee ${ }^{1,4}$, Hongyoon Choi ${ }^{5^{*}}$ and Hyung-Jun $\operatorname{Im}^{1 *}$ (D)

\begin{abstract}
Purpose: The linkage between the genetic and phenotypic heterogeneity of the tumor has not been thoroughly evaluated. Herein, we investigated how the genetic and metabolic heterogeneity features of the tumor are associated with each other in head and neck squamous cell carcinoma (HNSC). We further assessed the prognostic significance of those features.

Methods: The mutant-allele tumor heterogeneity (MATH) score $(n=508)$, a genetic heterogeneity feature, and tumor glycolysis feature (GlycoS) $(n=503)$ were obtained from the HNSC dataset in the cancer genome atlas (TCGA). We identified matching patients $(n=33)$ who underwent 18F-fluorodeoxyglucose positron emission tomography (FDG PET) from the cancer imaging archive (TCIA) and obtained the following information from the primary tumor: metabolic, metabolic-volumetric, and metabolic heterogeneity features. The association between the genetic and metabolic features and their prognostic values were assessed.
\end{abstract}

Results: Tumor metabolic heterogeneity and metabolic-volumetric features showed a mild degree of association with MATH ( $n=25, \rho=0.4 \sim 0.5, P<0.05$ for all features). The patients with higher FDG PET features and MATH died sooner. Combination of MATH and tumor metabolic heterogeneity features showed a better stratification of prognosis than MATH. Also, higher MATH and GlycoS were associated with significantly worse overall survival $(n=499, P=0.002$ and 0.0001 for MATH and GlycoS, respectively). Furthermore, both MATH and GlycoS independently predicted overall survival after adjusting for clinicopathologic features and the other ( $P=0.015$ and 0.006 , respectively).

Conclusion: Both tumor metabolic heterogeneity and metabolic-volumetric features assessed by FDG PET showed a mild degree of association with genetic heterogeneity in HNSC. Both metabolic and genetic heterogeneity features were predictive of survival and there was an additive prognostic value when the metabolic and genetic heterogeneity features were combined. Also, MATH and GlycoS were independent prognostic factors in HNSC; they can be used for precise prognostication once validated.

Keywords: ${ }^{18}$ F-fluorodeoxyglucose, Positron emission tomography, Heterogeneity, Radiogenomics, MATH

\footnotetext{
* Correspondence: chy1000@gmail.com; iiihjjj@gmail.com; http://tmtl.snu.ac.kr ${ }^{5}$ Department of Nuclear Medicine, Seoul National University Hospital, Seoul, Republic of Korea

${ }^{1}$ Department of Transdisciplinary Studies, Graduate School of Convergence Science and Technology, Seoul National University, Seoul, Republic of Korea Full list of author information is available at the end of the article
} 


\section{Introduction}

Cancer is a heterogenous disease at genetic, epigenetic, and phenotypic levels [1]. Cancer progression is driven by a genetic process of clonal evolution, which eventually causes tumor genetic heterogeneity, a tumor with multiple subsets of subclonal mutations [1]. Acquired tumor genetic heterogeneity is caused by the selective pressures during the evolution process and affected by tumor vasculature and immune system in the microenvironment [2]. Furthermore, genetic heterogeneity eventually drives the phenotypic heterogeneity of tumor by interacting environmental factors [3]. Heterogeneous subsets of tumor have different molecular targets, which may result in different levels of resistance to the cancer treatment [4]. Accordingly, tumor heterogeneity is associated with the progression and eventual clinical outcomes of cancer patients [5]. Thus, evaluation of tumor heterogeneity is crucial for selecting anticancer strategies and predicting clinical outcomes [6]. Advances in nextgeneration sequencing (NGS) have allowed for extensive understanding of tumor genetic heterogeneity and provided useful features to evaluate of tumor heterogeneity [7, 8]. The mutant allele tumor heterogeneity (MATH), a genetic heterogeneity feature, is easily calculated as a percentage of mutant allele frequencies among tumor-specific mutated loci. MATH has been known to have a prognostic value in HNSC and colon cancer $[9,10]$.

Phenotypical heterogeneity can be noninvasively studied using various imaging techniques including computed tomography $(\mathrm{CT})$, magnetic resonance imaging (MRI), and ${ }^{18} \mathrm{~F}$ fluorodeoxyglucose positron emission tomography (FDG PET) [11]. FDG PET is a compelling image modality to evaluate metabolic heterogeneity of tumors, a phenotypic tumor heterogeneity [12]. Recently, heterogeneity parameters obtained using FDG PET have been extensively evaluated and reported to have diagnostic and prognostic values in multiple types of malignancies including HNSC, nonsmall cell lung cancer, and pancreatic cancer [12-16]. Although the metabolic features evaluated by FDG PET are closely associated with biological factors in the tumor microenvironment $[12,17,18]$, it is still unknown whether metabolic heterogeneity is associated with genetic heterogeneity [14].

Herein, we investigated if metabolic heterogeneity based on FDG PET was associated with genetic heterogeneity represented by MATH. Furthermore, we explored the prognostic value of both metabolic and genetic heterogeneity features in predicting the outcomes of patients with HNSC.

\section{Materials and methods Data acquisition}

Genomic and clinical data were obtained from the head and neck squamous cell carcinoma dataset of the cancer genome atlas (TCGA-HNSC). The FDG PET data of the patients included in TCGA-HNSC was obtained from cancer imaging archive (TCIA) which is a publicly available repository. TCGA and TCIA data were acquired by a publicly available dataset that removed patient identifiers. The publicly available data were collected with patients' informed consent approved by the institutional review boards of all participating institutions following the 1964 Helsinki declaration and its later amendments or comparable ethical standards. A total of 528 clinical information which was updated at 2018/08/30 were acquired National Cancer Institute database for the survival analysis. The somatic variants data were acquired for 508 TCGA-HNSC patients from the NCI database using $\mathrm{R}$ data package 'TCGAmutations.' Glycolysis signature (Gly$\mathrm{coS}$ ) was previously assessed for the metabolic signatures of all TCGA samples [19] and downloaded from the website (http://choih.shinyapps.io/metabolicsignatures). In brief, GlycoS data were obtained by RNA sequencing data of TCGA samples by using gene set enrichment analysis and metabolic pathway genes of Reactome [20]. A total of 192 patients of TCGA-HNSC images in the TCIA matched with the genomic data of the TCGA were available. Finally, we identified 33 cases which included baseline FDG PET/CT scans and utilized the FDG PET scans for further analysis.

\section{Tumor metabolic features analyzed by FDG PET}

In this study, primary tumor segmentation of all FDG PET examinations was computed using PETedge tool of MIMvista (MIM Software Inc., USA) by an expert. Before calculating the texture feature, we have changed $X$, $Y, Z$ values of all data sets as the same values $(4.7,4.7$, 3.3) through trilinear voxel interpolation to compare each other. Then feature extraction was performed using LIFEx (IMIV, CEA, France) based on these regions of interest (ROIs) [21]. Maximum standardized uptake value (SUVmax), peak standardized uptake value (SUVpeak), metabolic tumor volume (MTV), and total lesion glycolysis (TLG) are the conventional metabolic or metabolic-volumetric parameters which are the most extensively studied in the previous studies and found to be prognostic in head and neck cancer [22-24]. Among the many heterogeneity parameters, we selected entropy and coefficient of variation (COV) because these features were reproducible and robust values in different reconstruction and acquisition time settings according to previous studies $[25,26]$. Two tumor metabolic features (SUVmax and SUVpeak), two metabolic-volumetric (TLG and MTV), and two metabolic heterogeneity features (entropy and COV) were obtained. Entropy was calculated based on SUV histogram using the equation; Entropy $=-\sum_{i} p(i) \times \log _{2}(p(i)+\varepsilon)$. Entropy reflects the randomness of the distribution where $\mathrm{p}(\mathrm{i})$ is the probability of occurrence of voxels with intensity $i$ and 
$\varepsilon=2 \mathrm{e}-16$. COV was calculated as standard deviation divided by SUVmean of the ROI. There were small tumors among the patients (range 0.36 3.93 $\mathrm{mL}$, median $1.14 \mathrm{~mL}$ ). However, we could calculate entropy and COV in all tumors, because entropy was the histogrambased parameter and COV can be calculated from SUV distribution. Twenty-five head and neck squamous cell carcinoma (HNSC) cases which have both FDG PET feature extraction data and gene mutation data were available to perform correlation analysis.

\section{Tumor genetic heterogeneity}

The MATH score was obtained as a percentage of the median absolute deviation (MAD) and median by clustering the variant allele frequency in each mutated loci using the $R$ 'maftools' package [27]. Each MATH score was calculated using MAF files for a total of 508 tumor samples, and this was used for survival analysis together with survival data.

\section{Survival analysis}

Overall survival (OS) can be obtained from the clinical data, which is defined as the period from the date of diagnosis until the date of death from any cause. The censored time is from date of initial diagnosis until the date of last contact (largest number of days) from all the clinical data files [28]. For evaluation of prognostic value of the features, we divided the patients into two groups (high and low groups) according to an optimized cut-off of each feature. The optimized cut-off was selected using the 'cutoff finder (http://molpath.charite.de/cutoff/index.jsp).' We selected the method for 'Survival: significance (log-rank test)' for cutoff determination. This cutoff is the most significant point from log-rank test which divide the variables into two groups. The high and low groups were compared using the log-rank test and Kaplan Meyer analysis. Cox regression analysis was also performed in multivariable survival analysis using continuous MATH, GlycoS, age, and categorical clinicopathologic variables (sex and tumor stage).

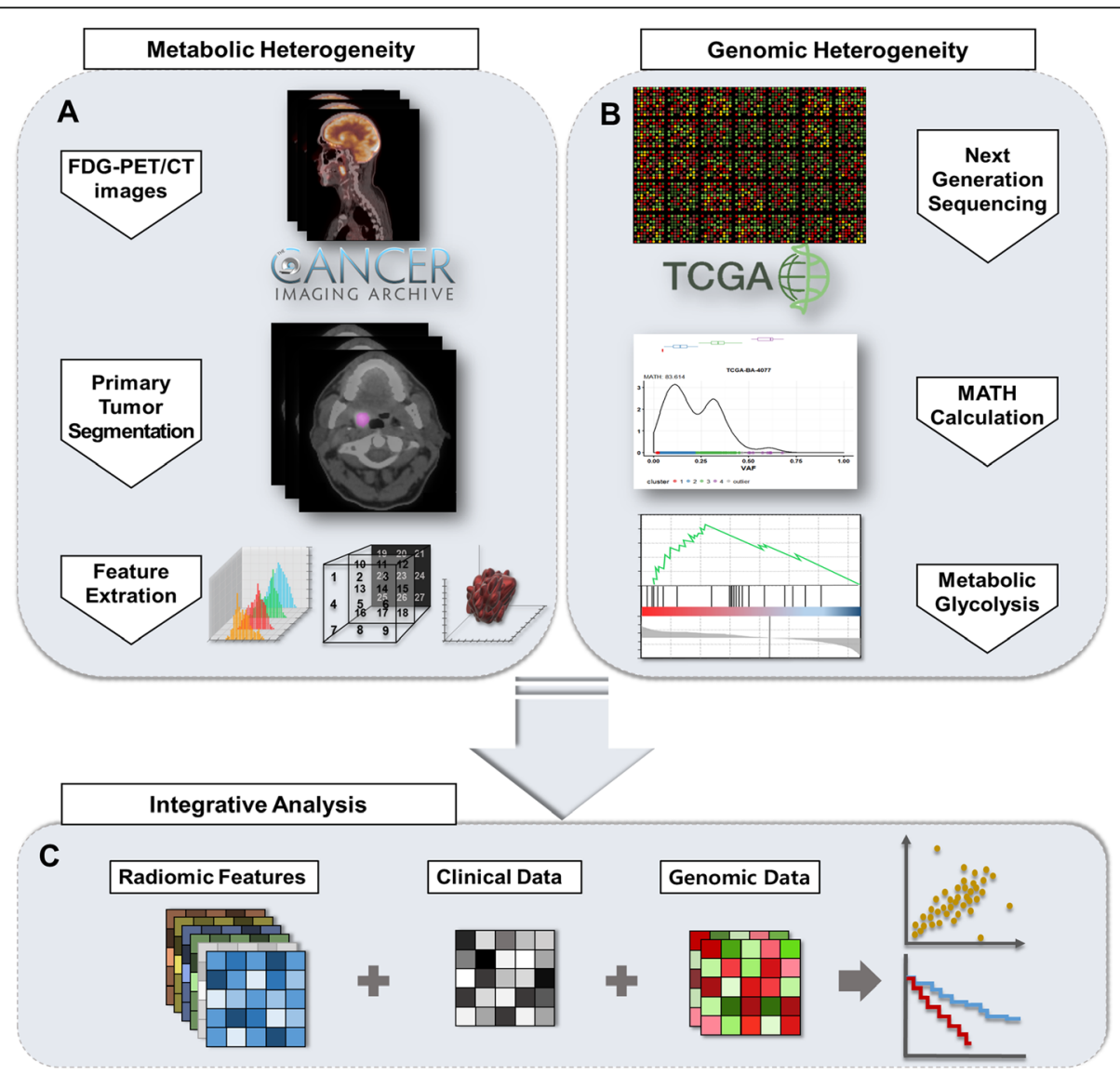

Fig. 1 Study scheme. A scheme for integrative study of radiogenomics. FDG PET data and genomic mutation data for TCGA-HNSC dataset were obtained from each database of TCIA and TCGA. a The primary tumor was manually assigned, and then ROls were computed for feature extraction. b MATH calculation using MAF files were done in R. Also, metabolic glycolysis (GlycoS) value was obtained using gene set enrichment analysis. c Clinical data of TCGA-HNSC was gained from TCGA. Total six features were selected and used for radiogenomic analysis. We statistically analyzed radiomic, clinical and genomic data using correlation analysis, Kaplan-Meier analysis, log-rank test 


\section{Statistical analysis}

Association between these genomic and tumor metabolic features was analyzed by using correlation analysis and the prognostic value of the parameters were assessed using the log-rank test. To analyze the correlation between two genomic and selected six FDG PET features, Spearman correlation analysis was performed. Correlation coefficients and $p$ values were gained and used to sort statistically significant features $(P$ value $<0.05)$. All statistical analyses were performed in R (version 3.4.4) and SPSS (version 25). All tests were two-sided and $P$ values less than 0.05 were considered significant.

\section{Results}

\section{Patient characteristics}

The scheme of this study is demonstrated in Fig. 1. The number of patients with genomic data was 508. The patients had a median age of 61 years (range of 20-90 years) and a median follow-up days of 633 days (range of 26417 days). Among them, 220 patients died during the follow-up. In all patients, $78 \%$ were stage III/IV, and there were an about three times higher number of men than women (371 vs. 137).

The characteristics of 25 patients who were available for both genomic and FDG PET analysis are summarized in Table 1. Primary tumor segmentation and feature extraction were performed using FDG PET scans of the patients. The median age of the patients was 56 years (range of 38-84 years). Among 25 patients with a median follow-up of 458 days (range of 30-6417 days), six patients died while 18 were alive.

\section{Association between genetic and FDG PET features}

Correlation analyses between MATH, GlycoS, and FDG PET features (SUVmax, SUVpeak, TLG, MTV, entropy, and $\mathrm{COV}$ ) were performed in 25 patients. Metabolic heterogeneity features and metabolic-volumetric features showed a trend for association with the genetic heterogeneity feature, MATH $(\rho=0.488, P=0.013$ for entropy; $\rho=0.402, P=0.047$ for COV $, \rho=0.521, P=0.008$ for MTV; $\rho=0.472, P=0.017$ for TLG) (Figs. 2 and 4). On the other hand, SUVmax and SUVpeak were not significantly associated with MATH $(\rho=0.328, P=0.110$ for SUVmax; $\rho=0.286, P=0.250$ for SUVpeak) (Fig. 2).

We evaluated the association between GlycoS calculated by gene expression and FDG PET features to use GlycoS as a surrogate of tumor metabolic features for more patients in survival analysis. TLG and MTV from FDG PET showed moderate degree of associations with the GlycoS ( $\rho=0.590, P=0.002$ for MTV; $\rho=0.570, P=$ 0.004 for TLG). The GlycoS showed a trend of positive correlation with entropy, while COV, SUVmax, and SUVpeak did not $(\rho=0.519, P=0.009$ for entropy; $\rho=$
Table 1 Patients characteristics (FDG-PET)

\begin{tabular}{|c|c|}
\hline Patients, $n$ & 25 (1 of not available clinical data) \\
\hline Median follow-up (days) & $458(30-6417)$ \\
\hline \multicolumn{2}{|l|}{ Vital status } \\
\hline Dead & 6 \\
\hline Alive & 18 \\
\hline \multicolumn{2}{|l|}{ Age (years) } \\
\hline Median & 56 \\
\hline Range & $38 \sim 84$ \\
\hline \multicolumn{2}{|l|}{ Gender } \\
\hline Male & 18 \\
\hline Female & 6 \\
\hline \multicolumn{2}{|l|}{ Clinical stage } \\
\hline 1 & 3 \\
\hline$\|$ & 5 \\
\hline III & 2 \\
\hline IVA & 13 \\
\hline IVB & 1 \\
\hline IVC & 0 \\
\hline \multicolumn{2}{|l|}{ Tumor site } \\
\hline Alveolar ridge & 1 \\
\hline Base of tongue & 2 \\
\hline Larynx & 5 \\
\hline Oral cavity & 4 \\
\hline Oral tongue & 3 \\
\hline Tonsil & 9 \\
\hline
\end{tabular}

0.393, $P=0.057$ for COV; $\rho=0.331, P=0.114$ for SUV$\max ; \rho=0.272, P=0.291$ for SUVpeak) (Fig. 3).

\section{Prognostic value of the genetic and FDG PET features}

We analyzed the prognostic value of genetic and FDG PET features. SUVmax, MTV, TLG, and entropy were predictive of OS $(P<0.05$ for the features), while COV tended to predict the OS $(P=0.072)$ (Figs. 4 and 5$)$. Also, MATH tended to predict the OS $(P=0.086)$ in the 25 patients. Thus, we tested the predictive value of using both heterogeneity features from genetic data and FDG PET. We divided the patients into two groups (low and high) based on both heterogeneity features. Low group consists of patients who are in low group for both MATH and FDG PET feature (COV or entropy) and high group consists of patients who are in high group for MATH and/or FDG PET feature (COV or entropy). We found that the combination of MATH and FDG heterogeneity features showed more robust predictive value of OS than using only MATH (MATH: $P=0.086$, MATH + COV: $P=0.024$, MATH + Entropy: $P=0.012$, Fig. 6 ). 

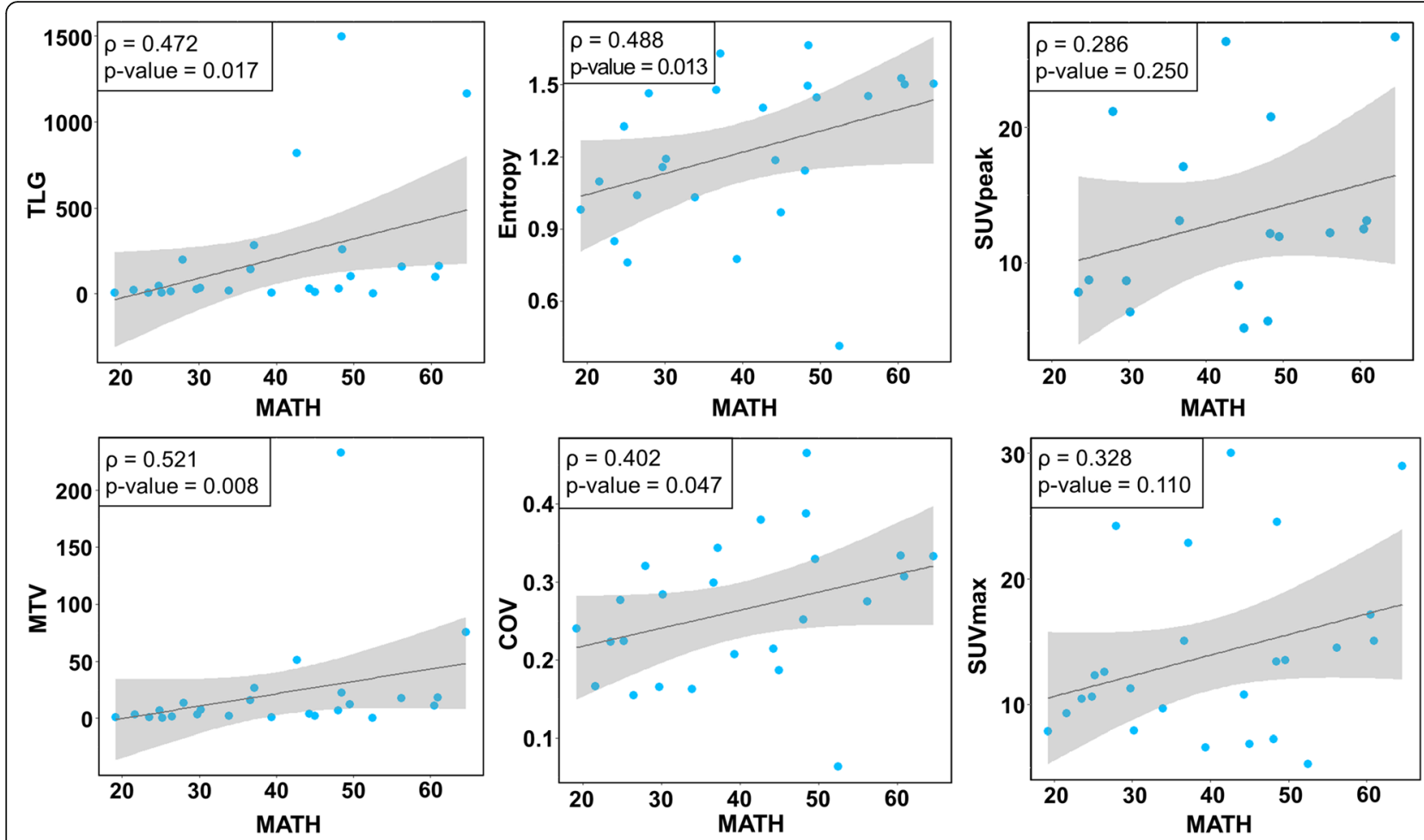

Fig. 2 Correlation between MATH and FDG PET features. Scatter plots for correlation analysis of MATH and FDG PET features. Each blue dots represent patients available for MATH and radiomic data $(N=25)$. Upper left box shows Spearman correlation coefficient $(\rho)$ and $P$ value. The dark gray line means a linear regression line and the gray region is $95 \%$ confidence region

Finally, we further analyzed the prognostic value of MATH and GlycoS in 499 patients. We found that MATH and GlycoS were highly predictive of OS in univariate analysis using log-rank test and Kaplan-Meyer analysis $(P=0.002$ for MATH; $P=0.0001$ for GlycoS) (Fig. 7a, b). MATH and GlycoS were predictive of OS even after adjustment using clinicopathologic features (age, sex, and tumor stage) in multivariate Cox regression analysis. Furthermore, both MATH and GlycoS were still significant prognostic factors even after including both features and the clinicopathologic features in the same model. This result indicates that both features have an additive role over each other to predict OS $(P=$ 0.015 for MATH; $P=0.006$ for GlycoS) (Table 2).

\section{Discussion}

We found that the tumor metabolic features estimated by FDG PET showed a mild but statistically significant level of association with tumor genetic heterogeneity. Specifically, tumor metabolic-volumetric and metabolic heterogeneity features of FDG PET were associated with MATH in a mild degree. This finding supports the notion that quantifiable FDG uptake features reflect the tumor heterogeneity at the genomic level in HNSC [10]. Also, there was additive prognostic value when the FDG
PET and genetic heterogeneity features were combined. Additionally, both genetic heterogeneity feature (MATH) and glycolysis feature (GlycoS) were independently predictive of OS even after adjusting for clinicopathologic features.

Recently, tumor imaging phenotypes were found to be related to gene expression profiles in HNSC [10, 17, 29-32]. Specifically, SUV and heterogeneity features estimated by FDG PET were related to 1177 differentially expressed genes in normal and tumor tissues [10]. Previous studies have shown a link between FDG uptake and several specific genes which modulate glucose metabolism [33-35]. Also, phenotypic whole tumor-level heterogeneity can be noninvasively recognized by various imaging techniques including computed tomography $(\mathrm{CT})$, magnetic resonance imaging (MRI), and FDG PET [11]. However, it has been unclear whether the genetic heterogeneity assessed by a small sample of tumor tissue can reflect the whole tumor-level phenotypic heterogeneity or not [6]. Also, there has been no study to evaluate the association of tumor heterogeneity measured by FDG PET and genomic analysis in patients with HNSC. As cancer cells are evolved in a heterogeneous spatiotemporal environment based on genetic heterogeneity, we hypothesized that genetic 

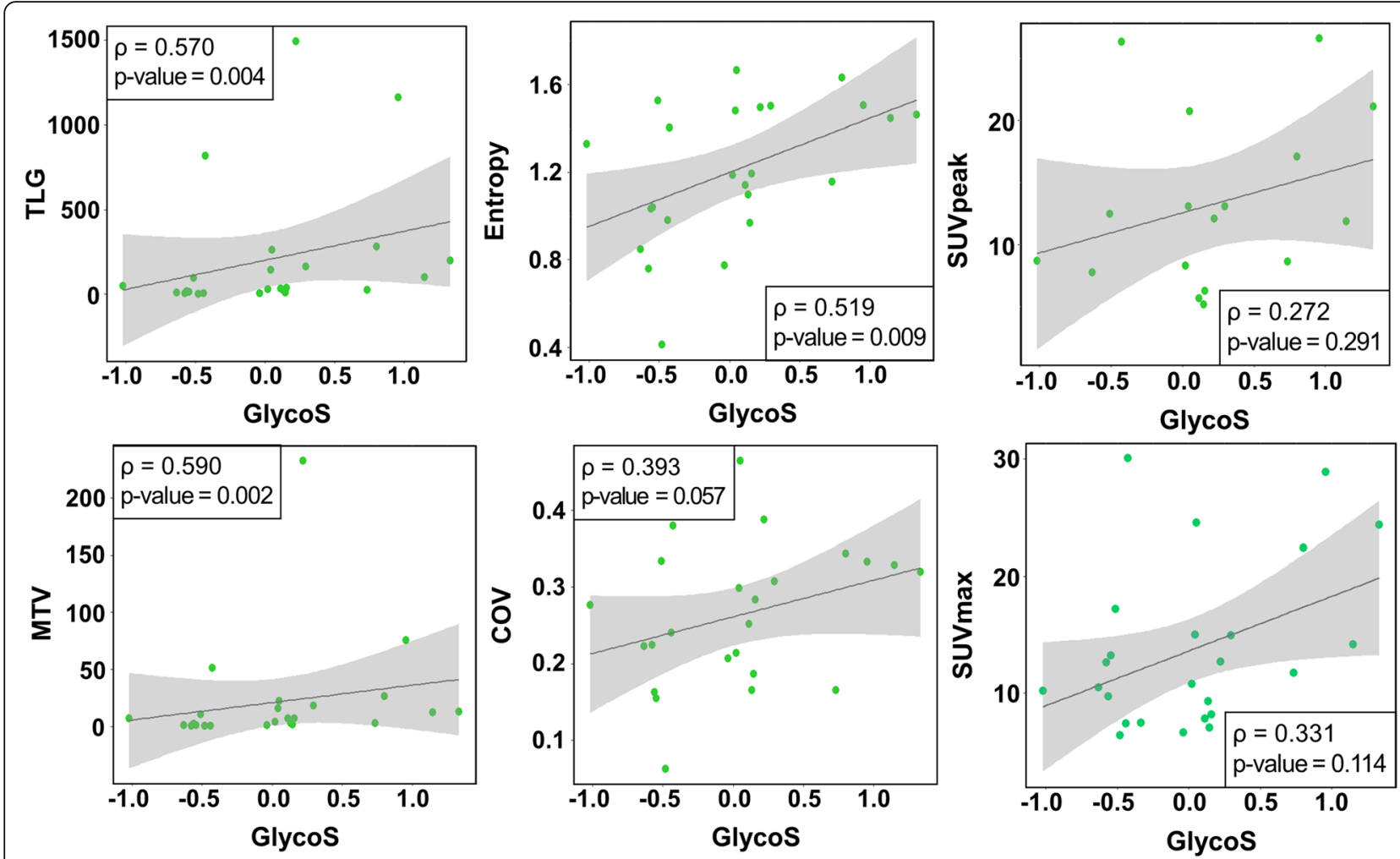

Fig. 3 Correlation between GlycoS and FDG PET features. Scatter plots for correlation analysis of GlycoS and FDG PET features. Each green dots represent patients available for MATH and radiomic data $(N=25)$. Upper left box shows Spearman correlation coefficient $(\rho)$ and $P$ value. The dark gray line means a linear regression line and the gray region is $95 \%$ confidence region

heterogeneity might be associated with whole tumor level heterogeneity measured by FDG PET. In this study, by utilizing the database of TCGA and TCIA, we were able to find that there is an association between whole tumor level heterogeneity based on FDG PET and genetic heterogeneity in HNSC. Although the association was statistically significant, the level of association was weak with correlation coefficients of $0.4 \sim 0.5$. This weak level of association was not a surprise because the methods to measure the tumor heterogeneity were totally different between FDG PET heterogeneity parameters and MATH. MATH was obtained from genetic sequencing data of a small portion of tumor, while FDG PET heterogeneity parameters were calculated from an imaging data reflecting metabolic status of a whole tumor area. It is noteworthy that there was a mild degree of association between MATH and FDG PET heterogeneity parameters, even with this striking difference of the methods to measure the tumor heterogeneity.

MATH is a genetic heterogeneity measure, which can be easily quantified as a percentage of mutant allele frequency among tumor-specific mutated loci. Also, the prognostic value of MATH has been validated in HNSC and colon cancer $[9,36,37]$. In the patients with HNSC, high MATH score was associated with increased mortality [36, 37]. Also, MATH was associated with the risk of metastases in patients with colon cancer [9]. However, the ability of MATH to represent tumor heterogeneity has not been tested by other modalities. We have demonstrated that MATH was highly associated with the representative heterogeneity features from FDG PET (entropy, COV). This result is in line with a recent study by Moon et al. They showed that Shannon's heterogeneity index was associated with entropy in patients with small cell lung cancer [38]. Furthermore, we found that MATH was predictive of OS in patients with HNSC even after adjusting clinicopathologic features.

Recent meta-analyses showed that various FDG PET features including SUVmax, MTV, and TLG were prognostic factors in multiple types of malignancies [39-42]. Also, heterogeneity features of FDG PET have shown to be associated with treatment response and clinical outcome in multiple types of malignancies [13, 15, 43-45]. Among the heterogeneity features, entropy and COV have been widely accepted and proven to be useful for predicting treatment response and clinical outcomes [13, 15, 44]. For example, entropy was predictive of OS in pancreatic cancer [15], and the 


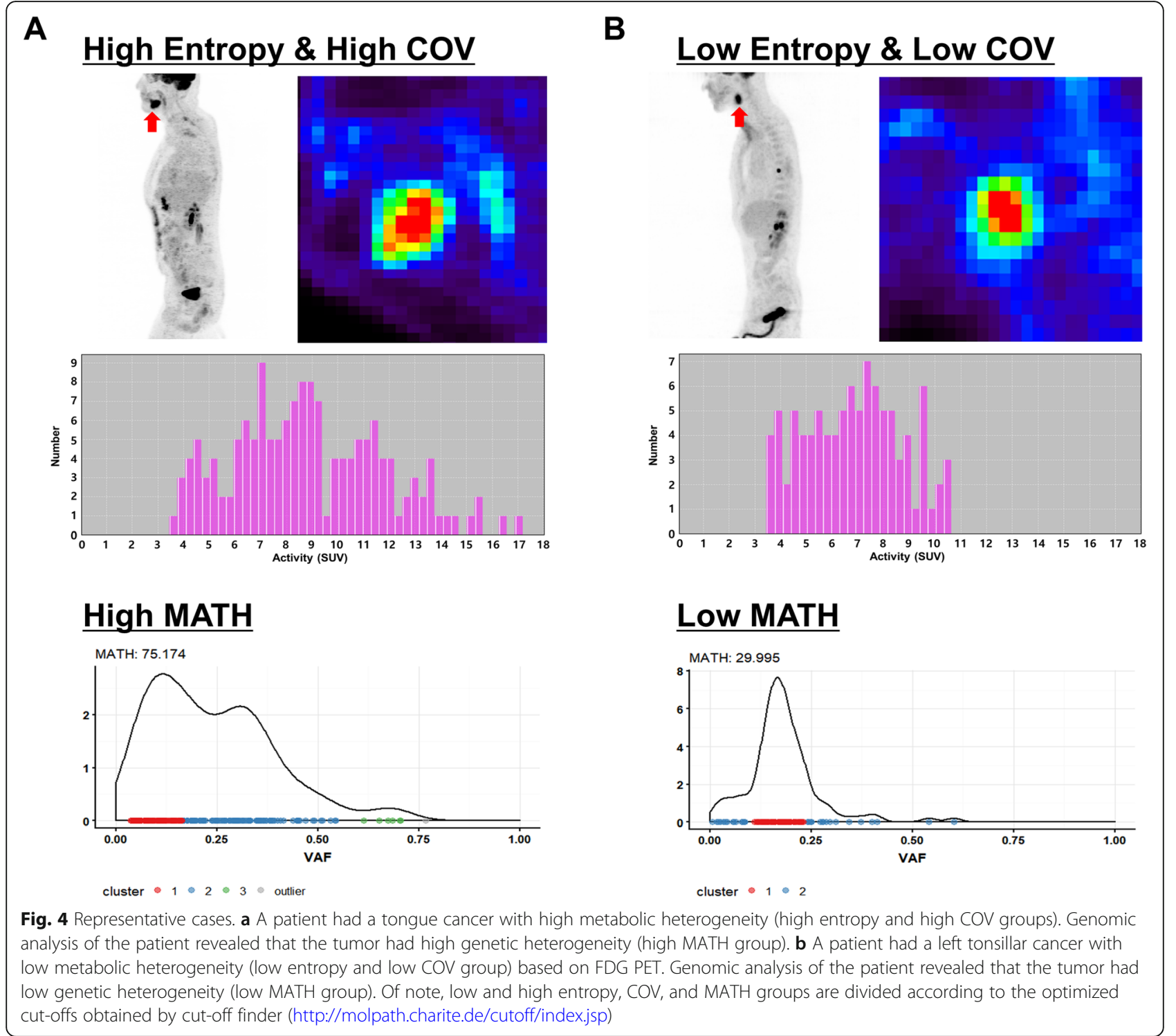

changes in entropy were independently associated with treatment response in erlotinib-treated non-small cell lung cancer [13]. Also, COV was superior to conventional parameters in predicting therapy response and disease progression in rectal cancer [44]. We found that entropy and $\mathrm{COV}$ were strongly associated with MATH, a genetic heterogeneity feature, which re-enforced the genetic background of the features and thus increased possibility of clinical utilization of the features.

MTV and TLG are radiomic features that represent metabolic-volumetric tumor burden. MTV is a measurement of tumor volume with increased glucose metabolism, while TLG is the product of MTV and the mean SUV of the volume. MTV and TLG are considered to be better prognostic factors than simple metabolic feature such as SUVmax [41, 46, 47]. In this study, we also found that MTV and TLG are significantly associated with genetic heterogeneity. As the tumor spatially grows, the larger volume of tumor likely to be more heterogenous reflecting genetic heterogeneity by cancer evolution. Multiple studies have shown that MTV and heterogeneity features of FDG PET such as COV and texture features are associated [48-50]. Therefore, tumor metabolic-volumetric features are likely to be an indicator of tumor genetic heterogeneity due to cancer evolution. Also, the association of MTV and TLG with genetic heterogeneity may further explain the robustness of the features in predicting the clinical outcomes.

Glycolysis is a crucial pathway regulating oncogenes, tumor suppressor genes, and glycolytic enzymes as well as accelerating cell proliferation in cellular metabolism [51]. Factors of metabolic glycolysis are associated with 


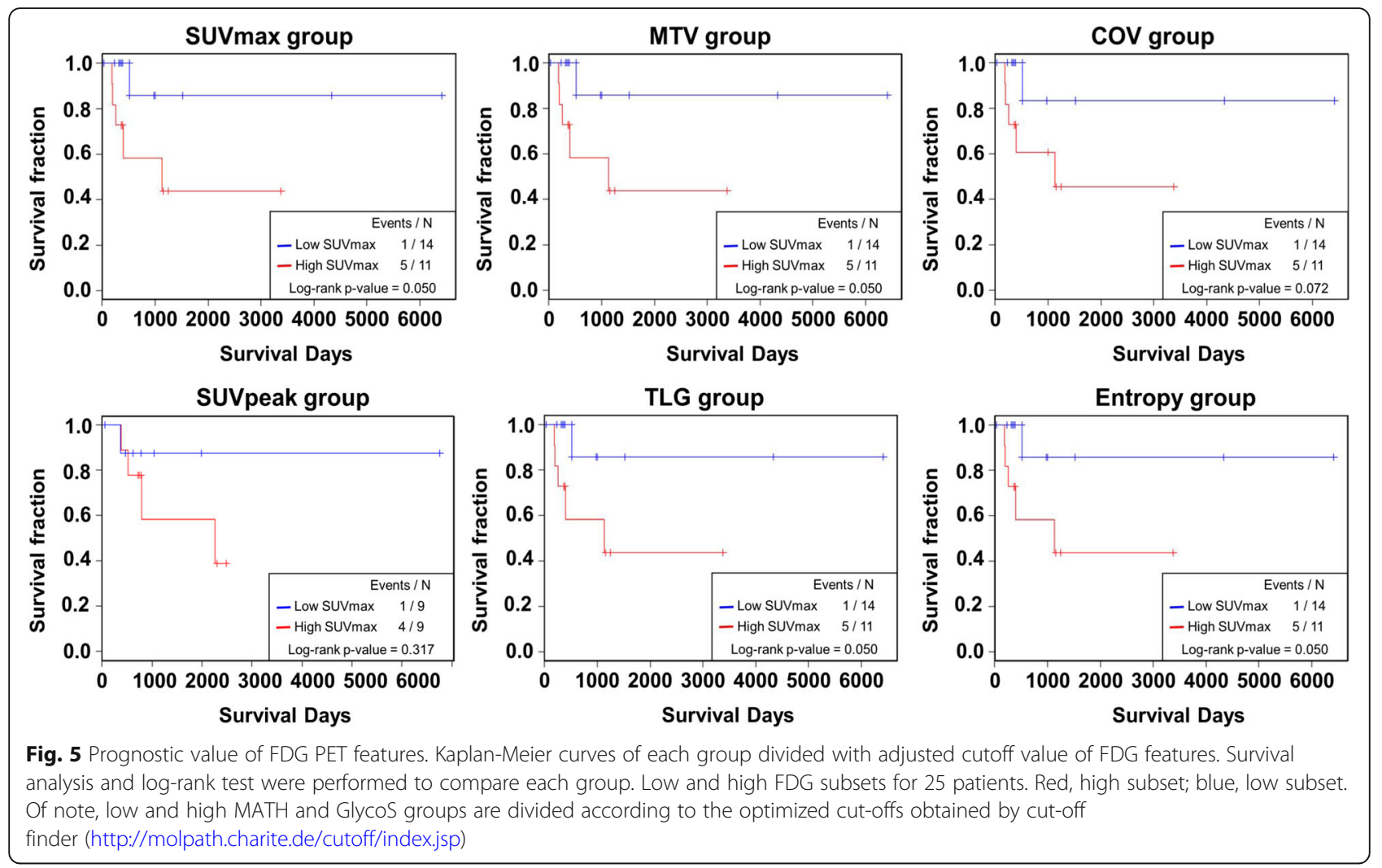

poor prognosis and tumor resistance to therapy in HNSC [52]. Also, glycolysis gene expression correlates FDG uptake features [30, 33-35]. However, the previous studies only explored the relationship of representative genes such as glucose transporter (GLUT) or hexokinase (HK). On the other hand, we utilized a novel glycolysis signature, GlycoS, which was derived from multiple glycolysis-associated genes defined by Reactome $[19,53]$. We found that metabolic-volumetric features (MTV, TLG) were significantly associated with GlycoS. Unexpectedly, SUVmax and SUVpeak were not associated with GlycoS. One potential explanation is that many glycolysisassociated genes may not influence the intensity of FDG uptake, because the FDG uptake kinetics is primarily determined by glucose transportation by GLUT and phosphorylation by HK [54]. Even though, a higher number of patients may prove the associations between SUVmax and GlycoS, since there was a trend of positive correlation $(P=$

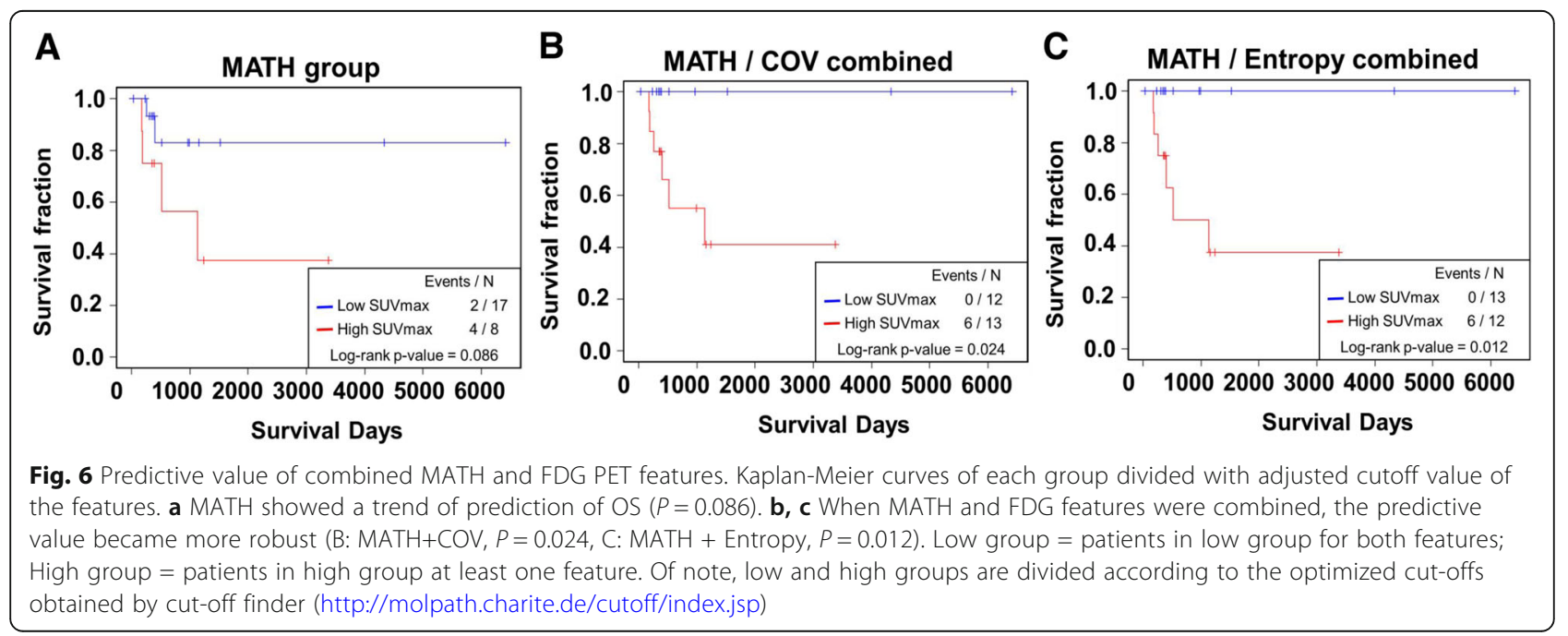




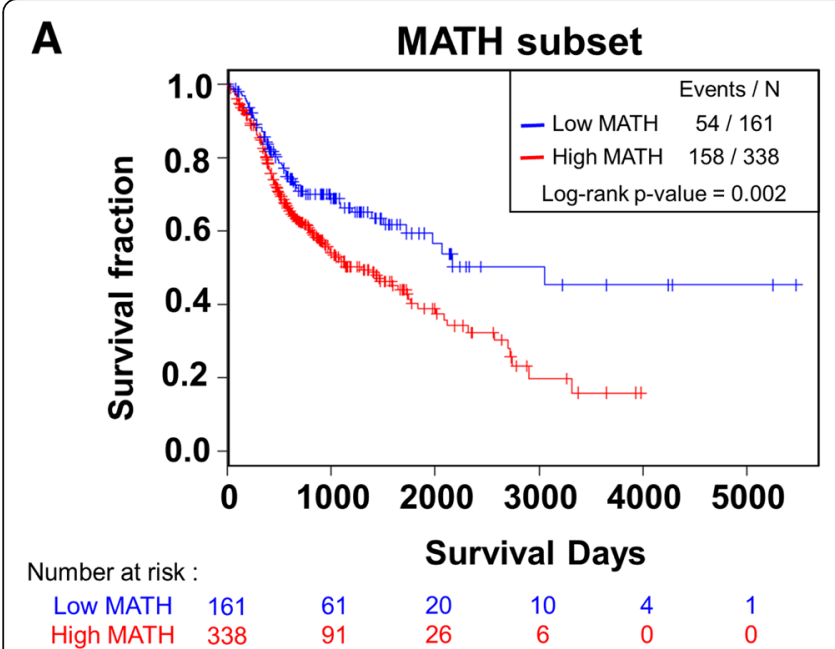

B

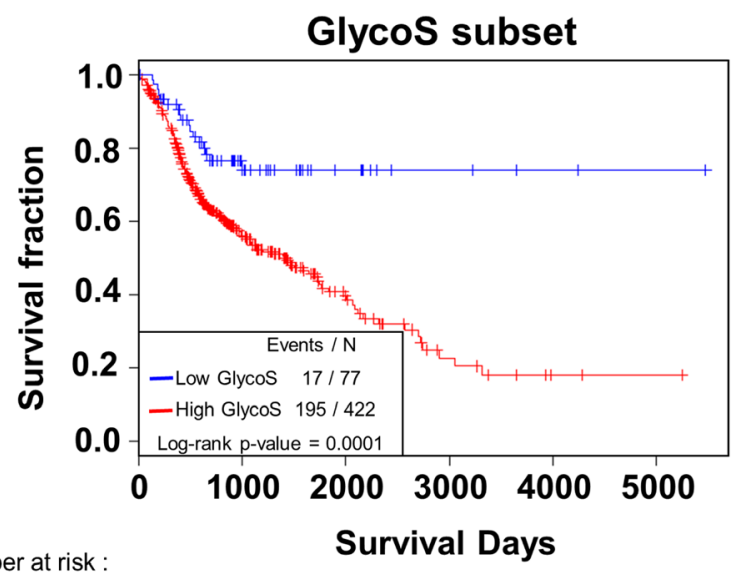

Number at risk:

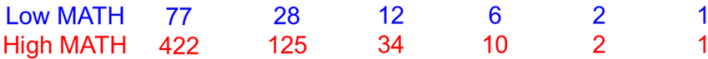

Fig. 7 Prognostic value of MATH and GlycoS. Kaplan-Meier curves of each group divided with adjusted cutoff value of genetic signatures. Survival analysis and log-rank test were performed to compare each group. a Low MATH and high MATH subsets for 499 patients. Red, high MATH (MATH > 37.17); blue, low MATH (MATH < 37.17). b Same analysis as (a) comparing low GlycoS and high GlycoS subsets. Red, high GlycoS (GlycoS > 0.80); blue, low GlycoS (GlycoS <0.80). Of note, low and high MATH and GlycoS groups are divided according to the optimized cut-offs obtained by cut-off finder (http://molpath.charite.de/cutoff/index.jsp)

0.114). Also, we found that GlycoS is predictive of OS in patients with HNSC. Furthermore, GlycoS was predictive of OS even after adjusting MATH and clinicopathologic features. This implies that GlycoS has an additive prognostic value over MATH.

There are several limitations to this study. First, a limited number of samples were available in public archives. Also, we found only a mild degree of association between the genetic and FDG PET heterogeneity, and there were large number of scatters outside of the standard deviation (Fig. 2). Further studies will yield clearer results if analyzed using a larger number of expanded data. Second, FDG PET data from TCIA were applied to different technologies, reconstruction, and attenuation correction methods. So each image is difficult to compare to each other, and even SUVmax values vary, which may affect clinical decision [55]. To solve this problem, we did voxel interpolation to make all images have uniform voxel sizes. Also, we used entropy and COV as a tumor heterogeneity texture features because these are the most reliable upon reconstruction method. Third, although metabolic features and genomic signatures obtained in this study were candidates for future biomarkers, these are not validated precisely. Although we used representative genomic and metabolic features which have clinical implications with prognosis, there might be better features than these eight features. In a future study, more features could be considered for understanding cause and effect through systemic tumor biology. Nonetheless, our results show a correlation between genetic heterogeneity features and metabolic heterogeneity features and prognostic value about each feature.

\section{Conclusion}

Tumor genetic heterogeneity was associated in a mild degree with metabolic heterogeneity measured by FDG PET in patients with HNSC. Genetic and metabolic heterogeneity features were predictive of OS, and there was additive prognostic value when the FDG PET and genetic heterogeneity features were combined. Moreover, genetic heterogeneity feature

Table 2 Multivariate cox regression test for MATH and GlycoS

\begin{tabular}{lll}
\hline & Hazard ratio (95\% Cl) & $P$ value \\
\hline MATH (unadjusted) & $1.013(1.002-1.024)$ & 0.016 \\
MATH (adjusted for age, gender, and tumor stage) & $1.016(1.004-1.027)$ & 0.007 \\
MATH (adjusted for GlycoS, age, gender, and tumor stage) & $1.014(1.003-1.026)$ & 0.015 \\
GlycoS (unadjusted) & $1.381(1.129-1.689)$ & 0.002 \\
GlycoS (adjusted for age, gender, and tumor stage) & $1.362(1.112-1.668)$ & 0.003 \\
GlycoS (adjusted for MATH, age, gender, and tumor stage) & $1.331(1.085-1.633)$ & 0.006 \\
\hline
\end{tabular}


(MATH) and glycolysis feature (GlycoS) were independent predictors of OS.

\section{Acknowledgements}

Not applicable.

\section{Authors' contributions}

$\mathrm{HC}$ and $\mathrm{HJ}$ conceptualized the study. JC and JAG did the statistical analysis. $\mathrm{JC}$ and $\mathrm{CO}$ wrote the manuscript. HL revised the manuscript. All authors read and approved the final manuscript.

\section{Funding}

This study was supported by the National Research Foundation of Korea (NRF) (NRF-2017R1D1A1B03035556, and NRF-2019M2D2A1A01058210), and the Ministry of Health and Welfare Korea (HI18C0886, and HI19C0339).

\section{Availability of data and materials}

All data analyzed during this study are included in this manuscript.

\section{Ethics approval and consent to participate}

All procedures performed in studies involving human participants were in accordance with the ethical standards of the institutional and/or national research committee and with the 1964 Helsinki declaration and its later amendments or comparable ethical standards. Informed consent was obtained from all individual participants included in the study.

\section{Consent for publication}

Consent has been obtained from participants to publish this work.

\section{Competing interests}

The authors declare that they have no competing interests.

\section{Author details}

'Department of Transdisciplinary Studies, Graduate School of Convergence Science and Technology, Seoul National University, Seoul, Republic of Korea. ${ }^{2}$ Radiation Medicine Research Institute, Seoul National University College of Medicine, Seoul, Republic of Korea. ${ }^{3}$ Department of Nuclear Medicine, Seoul ST. Mary's Hospital, Seoul, Republic of Korea. ${ }^{4}$ Department of Clinical Pharmacology and Therapeutics, Seoul National University College of Medicine and Hospital, Seoul, Republic of Korea. ${ }^{5}$ Department of Nuclear Medicine, Seoul National University Hospital, Seoul, Republic of Korea.

Received: 24 July 2019 Accepted: 24 September 2019

Published online: 21 November 2019

\section{References}

1. Dagogo-Jack I, Shaw AT. Tumour heterogeneity and resistance to cancer therapies. Nat Rev Clin Oncol. 2017;15:81-94.

2. Jamal-Hanjani M, Quezada SA, Larkin J, Swanton C. Translational implications of tumor heterogeneity. Clin Cancer Res. 2015;21:1258-66.

3. Marusyk A, Almendro V, Polyak K. Intra-tumour heterogeneity: a looking glass for cancer? Nat Rev Cancer. 2012;12:323-34.

4. Kleppe M, Levine RL. Tumor heterogeneity confounds and illuminates: assessing the implications. Nat Med. 2014;20:342-4.

5. McGranahan N, Swanton C. Clonal heterogeneity and tumor evolution: past, present, and the future. Cell. 2017;168:613-28.

6. Bedard PL, Hansen AR, Ratain MJ, Siu LL. Tumour heterogeneity in the clinic. Nature. 2013;501:355-64.

7. Navin N, Kendall J, Troge J, Andrews P, Rodgers L, Mclndoo J, et al. Tumour evolution inferred by single-cell sequencing. Nature. 2011;472:90-4.

8. Jacoby MA, Duncavage EJ, Walter MJ. Implications of tumor clonal heterogeneity in the era of next-generation sequencing. Trends Cancer. 2015;1:231-41.

9. Rajput A, Bocklage T, Greenbaum A, Lee J-H, Ness SA. Mutant-allele tumor heterogeneity scores correlate with risk of metastases in colon cancer. Clin Colorectal Cancer. 2017;16:e165-e70.

10. Tixier F, Cheze-Le Rest C, Chezeaud S, Key S, Simon B, Potard G, et al. FDG PET derived quantitative heterogeneity features reflect gene expression profiles in head and neck cancer. J Nucl Med. 2014;55:450.

11. Lambin P, Leijenaar RTH, Deist TM, Peerlings J, de Jong EEC, van Timmeren $J$, et al. Radiomics: the bridge between medical imaging and personalized medicine. Nat Rev Clin Oncol. 2017;14:749-62.
12. Chicklore S, Goh V, Siddique M, Roy A, Marsden PK, Cook GJ. Quantifying tumour heterogeneity in 18F-FDG PET/CT imaging by texture analysis. Eur J Nucl Med Mol Imaging. 2013;40:133-40.

13. Cook GJ, O'Brien ME, Siddique M, Chicklore S, Loi HY, Sharma B, et al. Nonsmall cell lung cancer treated with Erlotinib: heterogeneity of (18) F-FDG uptake at PET-association with treatment response and prognosis. Radiology. 2015;276:883-93.

14. Hatt M, Tixier F, Pierce L, Kinahan PE, Le Rest CC, Visvikis D. Characterization of PET/CT images using texture analysis: the past, the present ... any future? Eur J Nucl Med Mol Imaging. 2017;44:151-65.

15. Hyun SH, Kim HS, Choi SH, Choi DW, Lee JK, Lee KH, et al. Intratumoral heterogeneity of (18) F-FDG uptake predicts survival in patients with pancreatic ductal adenocarcinoma. Eur J Nucl Med Mol Imaging. 2016;43:1461-8.

16. Folkert MR, Setton J, Apte AP, Grkovski M, Young RJ, Schoder H, et al. Predictive modeling of outcomes following definitive chemoradiotherapy for oropharyngeal cancer based on FDG-PET image characteristics. Phys Med Biol. 2017;62:5327-43

17. Na K, Choi H. Tumor metabolic features identified by 18F-FDG PET correlate with gene networks of immune cell microenvironment in head and neck cancer. J Nucl Med. 2018;59:31-7.

18. Choi $\mathrm{H}, \mathrm{Na} \mathrm{K}$. Integrative analysis of imaging and transcriptomic data of the immune landscape associated with tumor metabolism in lung adenocarcinoma: clinical and prognostic implications. Theranostics. 2018;8:1956.

19. Choi H, Na K. Pan-cancer analysis of tumor metabolic landscape associated with genomic alterations. Mol Cancer. 2018;17:150.

20. Joshi-Tope G, Gillespie M, Vastrik I, D'Eustachio P, Schmidt E, de Bono B, et al. Reactome: a knowledgebase of biological pathways. Nucleic Acids Res. 2005:33:D428-D32.

21. Nioche C, Orlhac F, Boughdad S, Reuze S, Goya-Outi J, Robert C, et al. LIFEx: a freeware for Radiomic feature calculation in multimodality imaging to accelerate advances in the characterization of tumor heterogeneity. Cancer Res. 2018:78:4786-9.

22. Han S, Kim YJ, Woo S, Suh CH, Lee JJ. Prognostic value of volumetric parameters of pretreatment 18F-FDG PET/CT in esophageal cancer: a systematic review and meta-analysis. Clin Nucl Med. 2018;43:887-94.

23. Torizuka T, Tanizaki Y, Kanno T, Futatsubashi M, Naitou K, Ueda Y, et al. Prognostic value of 18F-FDG PET in patients with head and neck squamous cell cancer. AJR Am J Roentgenol. 2009:192:W156-W60.

24. Wang L, Bai J, Duan P. Prognostic value of 18F-FDG PET/CT functional parameters in patients with head and neck cancer: a meta-analysis. Nucl Med Commun. 2019;40:361-9.

25. Bailly C, Bodet-Milin C, Couespel S, Necib H, Kraeber-Bodéré F, Ansquer C, et al. Revisiting the robustness of PET-based textural features in the context of multi-centric trials. PLoS One. 2016:11:e0159984.

26. Forgacs A, Jonsson HP, Dahlbom M, Daver F, DiFranco MD, Opposits G, et al. A study on the basic criteria for selecting heterogeneity parameters of F18-FDG PET images. PLoS One. 2016;11:e0164113.

27. Mayakonda A, Lin D-C, Assenov Y, Plass C, Koeffler HP. Maftools: efficient and comprehensive analysis of somatic variants in cancer. Genome Res. 2018;28:1747-56.

28. Liu J, Lichtenberg T, Hoadley KA, Poisson LM, Lazar AJ, Cherniack AD, et al. An integrated TCGA Pan-cancer clinical data resource to drive high-quality survival outcome analytics. Cell. 2018:173:400-16.

29. Chen R-Y, Lin Y-C, Shen W-C, Hsieh T-C, Yen K-Y, Chen S-W, et al. Associations of tumor PD-1 ligands, Immunohistochemical studies, and textural features in 18F-FDG PET in squamous cell carcinoma of the head and neck. Sci Rep. 2018:8:105.

30. Wilson GD, Thibodeau BJ, Fortier LE, Pruetz BL, Galoforo S, Baschnagel AM, et al. Glucose metabolism gene expression patterns and tumor uptake of 18F-Fluorodeoxyglucose after radiation treatment. Int J Radiat Oncol Biol Phys. 2014;90:620-7.

31. Nair VS, Gevaert O, Davidzon G, Plevritis SK, West R. NF-KB protein expression associates with 18F-FDG PET tumor uptake in non-small cell lung cancer: a radiogenomics validation study to understand tumor metabolism. Lung Cancer. 2014;83:189-96.

32. Hong EK, Choi SH, Shin DJ, Jo SW, Yoo R-E, Kang KM, et al. Radiogenomics correlation between MR imaging features and major genetic profiles in glioblastoma. Eur Radiol. 2018:28:4350-61.

33. Tohma T, Okazumi S, Makino H, Cho A, Mochiduki R, Shuto K, et al. Relationship between glucose transporter, hexokinase and FDG-PET in esophageal cancer. Hepatogastroenterology. 2005;52:486-90. 
34. Hamada K, Tomita Y, Qiu Y, Zhang B, Ueda T, Myoui A, et al. 18 F-FDG-PET of musculoskeletal tumors: a correlation with the expression of glucose transporter 1 and hexokinase II. Ann Nucl Med. 2008;22:699-705.

35. Higashi T, Saga T, Nakamoto $Y$, Ishimori T, Mamede MH, Wada M, et al. Relationship between retention index in dual-phase 18F-FDG PET, and hexokinase-II and glucose transporter-1 expression in pancreatic cancer. J Nucl Med. 2002;43:173-80.

36. Mroz EA, Tward AD, Hammon RJ, Ren Y, Rocco JW. Intra-tumor genetic heterogeneity and mortality in head and neck cancer: analysis of data from the cancer genome atlas. PLoS Med. 2015;12:e1001786.

37. Mroz EA, Tward AD, Pickering CR, Myers JN, Ferris RL, Rocco JW. High intratumor genetic heterogeneity is related to worse outcome in patients with head and neck squamous cell carcinoma. Cancer. 2013;119: 3034-42.

38. Moon SH, Kim J, Joung J-G, Cha H, Park W-Y, Ahn JS, et al. Correlations between metabolic texture features, genetic heterogeneity, and mutation burden in patients with lung cancer. Eur J Nucl Med Mol Imaging. 2019;46: 446-54

39. Na F, Wang J, Li C, Deng L, Xue J, Lu Y. Primary tumor standardized uptake value measured on F18-Fluorodeoxyglucose positron emission tomography is of prediction value for survival and local control in nonsmall-cell lung cancer receiving radiotherapy: meta-analysis. J Thorac Oncol. 2014;9:834-42.

40. Sarker A, Im HJ, Cheon GJ, Chung HH, Kang KW, Chung JK, et al. Prognostic implications of the SUVmax of primary tumors and metastatic lymph node measured by 18F-FDG PET in patients with uterine cervical cancer: a metaanalysis. Clin Nucl Med. 2016:41:34-40.

41. Im HJ, Kim TS, Park SY, Min HS, Kim JH, Kang HG, et al. Prediction of tumour necrosis fractions using metabolic and volumetric 18F-FDG PET/CT indices, after one course and at the completion of neoadjuvant chemotherapy, in children and young adults with osteosarcoma. Eur J Nucl Med Mol Imaging. 2012:39:39-49.

42. Pak K, Cheon GJ, Nam HY, Kim SJ, Kang KW, Chung JK, et al. Prognostic value of metabolic tumor volume and Total lesion glycolysis in head and neck cancer: a systematic review and meta-analysis. J Nucl Med. 2014;55: 884-90.

43. Werner RA, Bundschuh RA, Higuchi T, Javadi MS, Rowe SP, Zsótér N, et al. Volumetric and texture analysis of pretherapeutic 18F-FDG PET can predict overall survival in medullary thyroid cancer patients treated with Vandetanib. Endocrine. 2019;63:293-300.

44. Bundschuh RA, Dinges J, Neumann L, Seyfried M, Zsoter N, Papp L, et al. Textural parameters of tumor heterogeneity in $(1,8)$ F-FDG PET/CT for therapy response assessment and prognosis in patients with locally advanced rectal cancer. J Nucl Med. 2014;55:891-7.

45. O'Connor JP, Rose CJ, Waterton JC, Carano RA, Parker GJ, Jackson A. Imaging intratumor heterogeneity: role in therapy response, resistance, and clinical outcome. Clin Cancer Res. 2015;21:249-57.

46. Im HJ, Kim YK, Kim Yl, Lee JJ, Lee WW, Kim SE. Usefulness of combined metabolic-volumetric indices of (18) F-FDG PET/CT for the early prediction of Neoadjuvant chemotherapy outcomes in breast cancer. Nucl Med Mol Imaging. 2013:47:36-43.

47. Lee JW, Kang CM, Choi HJ, Lee WJ, Song SY, Lee JH, et al. Prognostic value of metabolic tumor volume and total lesion glycolysis on preoperative 18F-FDG PET/CT in patients with pancreatic cancer. J Nucl Med. 2014;55:898-904.

48. Hatt M, Majdoub M, Vallières M, Tixier F, Le Rest CC, Groheux D, et al. 18FFDG PET uptake characterization through texture analysis: investigating the complementary nature of heterogeneity and functional tumor volume in a multi-cancer site patient cohort. J Nucl Med. 2015;56:38-44.

49. Orlhac F, Soussan M, Maisonobe J-A, Garcia CA, Vanderlinden B, Buvat I. Tumor texture analysis in 18F-FDG PET: relationships between texture parameters, histogram indices, standardized uptake values, metabolic volumes, and total lesion glycolysis. J Nucl Med. 2014;55:414-22.

50. Hatt M, Cheze-le Rest C, Van Baardwijk A, Lambin P, Pradier O, Visvikis D. Impact of tumor size and tracer uptake heterogeneity in (18) F-FDG PET and CT non-small cell lung cancer tumor delineation. J Nucl Med. 2011; 52:1690-7.

51. Cairns RA, Harris IS, Mak TW. Regulation of cancer cell metabolism. Nat Rev Cancer. 2011;11:85.

52. Kumar D. Regulation of glycolysis in head and neck squamous cell carcinoma. Postdoc J. 2017;5:14-28.
53. Croft D, Mundo AF, Haw R, Milacic M, Weiser J, Wu G, et al. The Reactome pathway knowledgebase. Nucleic Acids Res. 2014;42:D472-7.

54. Haberkorn U, Ziegler SI, Oberdorfer F, Trojan H, Haag D, Peschke P, et al. FDG uptake, tumor proliferation and expression of glycolysis associated genes in animal tumor models. Nucl Med Biol. 1994;21:827-34.

55. Schoder H, Erdi YE, Chao K, Gonen M. Clinical implications of different image reconstruction parameters for interpretation of whole-body PET studies in cancer patients. J Nucl Med. 2004;45:559-66.

\section{Publisher's Note}

Springer Nature remains neutral with regard to jurisdictional claims in published maps and institutional affiliations.

\section{Submit your manuscript to a SpringerOpen ${ }^{\circ}$ journal and benefit from:}

- Convenient online submission

- Rigorous peer review

- Open access: articles freely available online

High visibility within the field

- Retaining the copyright to your article

Submit your next manuscript at $\boldsymbol{\nabla}$ springeropen.com 